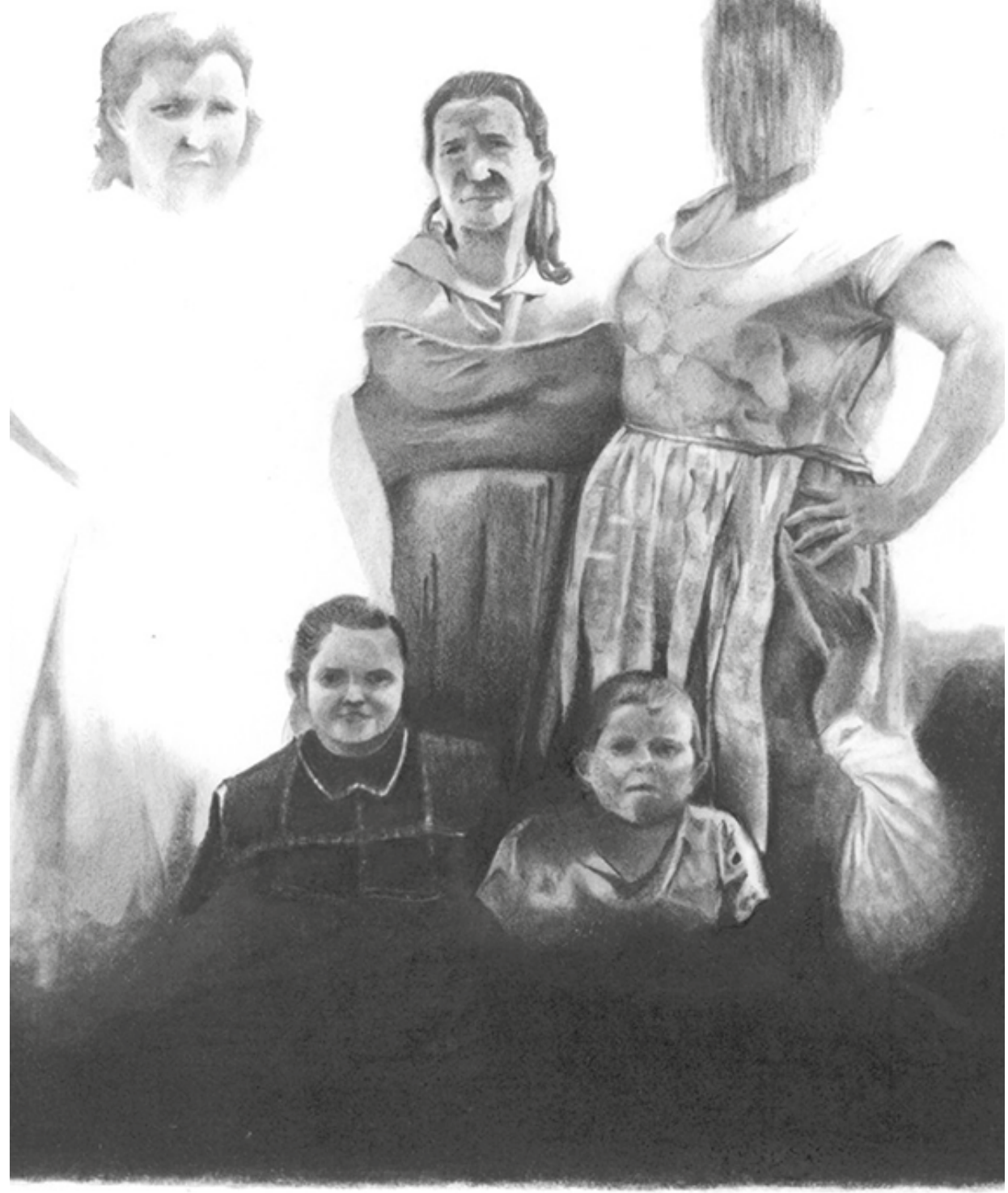

Luis Fernando Salazar Espinosa

Sin título

De la serie Psiquis

Lápiz sobre papel

$18 \times 15 \mathrm{~cm}$

2017

Medellín 


\title{
El marco jurídico del enfoque diferencial en políticas públicas para mujeres indígenas en Colombia*
}

\author{
Isabela Figueroa (Brasil) ${ }^{* *}$ \\ Noriana Marcela Franco Novoa (Colombia) ${ }^{* * *}$
}

\section{Resumen}

Ese artículo revisa algunas de las múltiples violencias sufridas por las mujeres indígenas víctimas del conflicto armado en Colombia, así como algunas de las políticas públicas del Estado orientadas a disminuir su situación de vulnerabilidad. Una de las herramientas de políticas públicas para la atención a grupos minoritarios es el Ilamado «enfoque diferencial». Ese artículo busca identificar el uso que distintos organismos del Estado han hecho de ese término - como la Corte Constitucional lo viene comprendiendo- y la relación de su aplicación con la participación de las mujeres indígenas en el diseño e implementación de políticas públicas. A partir de una revisión documental de doctrina, leyes y revisión jurisprudencial de casos nacionales e internacionales se espera contribuir a la discusión, creación y ejecución de medidas especiales que garanticen el pleno ejercicio de los derechos de los pueblos indígenas. Se concluye que el Estado colombiano no ha incluido a las mujeres indígenas en su planificación, vigilancia e implementación de las políticas públicas con perspectivas étnicas y de género.

\section{Palabras clave}

Políticas Públicas; Enfoque Diferencial; Género; Etnia; Derechos Humanos.

\footnotetext{
* Este artículo de reflexión presenta algunos avances de la investigación intitulada Alcance del enfoque diferencial en la implementación del Acuerdo de Paz de Colombia en torno a la garantía de los derechos colectivos de las comunidades étnicas, Universidad del Magdalena.

** Abogada. Magíster en Derecho Económico. Magíster en Derecho y Política de los Pueblos Indígenas. Magíster en Derecho Aborigen. Doctorada en Estudios Culturales Latinoamericanos. Posdoctorada en Política Social. Profesora e investigadora de la Universidad del Magdalena, Colombia. Correo electrónico: ifigueroa@unimagdalena.edu.co - Orcid: 0000-0002-9119-3615 Google Scholar: https://scholar.google.es/citations?hl=es\&user=fcvux-wAAAAJ

*** Abogada. Candidata a magíster en la Universidad del Colorado, Estados Unidos. Correo electrónico: nofr3398@colorado.edu
} 


\title{
Cómo citar este artículo
}

Figueroa, Isabela y Franco Novoa, Noriana Marcela. (2020). El marco jurídico del enfoque diferencial en políticas públicas para mujeres indígenas en Colombia. Estudios Políticos (Universidad de Antioquia), 57, pp. 71-90. DOI: 10.17533/udea. espo.n57a04

\section{The Legal Framework of the Differential Approach in Public Policies for Indigenous Women in Colombia}

\begin{abstract}
This article reviews some of the multiple violences suffered by indigenous women victims of the armed conflict in Colombia, as well as some of the State's public policies aimed at reducing their vulnerability. One of the administrative tools for attention to minority groups is the so-called «differential approach». This article seeks to identify the use that different State agencies have made of the term, how the Constitutional Court has been conceiving it, and the relationship between its application and the participation of indigenous women in the design and implementation of public policies. From a literature review of doctrines and laws, and a jurisprudential review of national and international cases, this study

[ 72 ] hopes to contribute to the discussion, creation, and execution of special measures that guarantee the full exercise of the rights of indigenous peoples. The analysis concludes that the Colombian State has not included indigenous women in its planning, monitoring, and implementation of public policies with ethnic and gender perspectives.
\end{abstract}

\section{Keywords}

Public Politics; Differential Approach; Gender; Ethnicity; Human Rights. 
El marco jurídico del enfoque diferencial en políticas públicas para mujeres indígenas...

\section{Introducción}

En 2006 la Comisión Interamericana de Derechos Humanos (CIDH) aseguró que la situación de vulnerabilidad de las mujeres indígenas en Colombia es especialmente crítica. Sobre ellas recaen, además de los graves efectos del conflicto armado, una larga historia de discriminación y exclusión por su condición de mujeres e indígenas $(\mathrm{CIDH}, 2006,18$ de octubre a, p. 47). De hecho, desde la creación del Estado colombiano las mujeres indígenas son triplemente discriminadas, su vulnerabilidad es determinada por la concordancia de factores en razón de género, etnia y condición social (Observatorio de Asuntos de Género, 2013). Esa constelación de factores, a su vez, expone a las mujeres indígenas a numerosas situaciones que ponen en peligro su identidad física, psicológica y cultural.

Lideresas de las diferentes comunidades indígenas de Colombia testificaron la unión directa entre la salvaguarda de sus derechos y el disfrute pleno y libre de sus tierras y territorios ancestrales, por lo que la protección de sus derechos de posesión y propiedad es requisito para la protección de su integridad física y moral (CIDH, 2006, 18 de octubre a). En 2011 las mujeres indígenas fueron víctimas de diferentes grupos armados, sufriendo abusos por parte de «agentes del Estado, miembros de grupos paramilitares post desmovilización e integrantes de las FARC-EP y del Ejército de Liberación Nacional» (CIDH, 2011, 31 de diciembre, p. 12). En efecto, debido a la proximidad de sus territorios a las zonas álgidas del conflicto, los pueblos tradicionales condensan el grupo poblacional más afectado por el conflicto y el más desamparado por el Estado en el contexto del conflicto armado interno (CIDH, 2011, 31 de diciembre).

La identificación y sistematización de los obstáculos específicos que afrontan las mujeres indígenas en Colombia requiere la creación de políticas públicas aplicables a su situación en el marco del posconflicto. Diversos programas y políticas públicas gubernamentales y de agencias de cooperación usan, cada vez más, la noción de enfoque diferencial en la aplicación de medidas de protección de derechos a grupos más vulnerables. La Corte Constitucional colombiana también ha emitido fallos refiriéndose a ese enfoque. Más relevante aún, «enfoque diferencial» es un lenguaje utilizado enfáticamente en el Acuerdo de Paz entre el Estado colombiano y las Fuerzas Armadas Revolucionarias de Colombia-Ejército del Pueblo (FARC-EP). 
La utilización de un enfoque distinto en la aplicación de políticas públicas no puede estar vacío de contenido jurídico, especialmente porque pretende fortalecer la protección de los derechos humanos de grupos vulnerables en el ámbito del posconflicto. ¿Cuáles son, entonces, las implicancias jurídicas del llamado enfoque diferencial? ¿Políticas públicas con enfoque diferencial efectivamente ofrecen mayor protección a los derechos del grupo destinatario?

Este artículo presenta algunos avances de una investigación cualitativa que, con base a una revisión documental de doctrina, leyes y revisión jurisprudencial de casos nacionales e internacionales, y en entrevistas semiestructuradas con líderes y lideresas indígenas y afrocolombianas de los departamentos del Magdalena y del Cauca, se analiza el uso y la aplicación de la noción de enfoque diferencial, presentando algunas de las reflexiones relacionadas con políticas hacia las mujeres indígenas. Los programas, sentencias y leyes revisadas en ese texto no son de ningún modo exhaustivas, sino que proponen indagar sobre las pretensiones y alcances del llamado «enfoque diferencial».

\section{Situación de las mujeres indígenas en Colombia}

\subsection{Múltiple vulnerabilidad: género, etnia y desplazamiento}

Pertenecer a un pueblo indígena es estar expuesto a una situación continua de vulnerabilidad manifiesta, derivada de la discriminación, la ignorancia y la desinformación. En América Latina los indígenas conforman $14 \%$ de las personas en situación de pobreza y 17\% de las personas en situación de extrema pobreza. Alrededor de $43 \%$ de las familias indígenas de América Latina son afectadas por la pobreza y $24 \%$ están en situación de extrema pobreza (Ministerio de Salud y Protección Social, 2016). Esa vulnerabilidad se sobredimensiona en el marco de un conflicto armado, especialmente para las mujeres indígenas que pasan a ser un blanco de agentes armados, estatales y no estatales en su lucha por control territorial (CIDH, 2011, 28 de diciembre, p. 27).

Uno de los resultados deseados de las acciones de los agentes armados son los desplazamientos de las comunidades tradicionales de sus tierras, lo que agrava aún más la situación de esas mujeres, tal como reconoció la Corte Constitucional (Auto 092 del 14 de abril de 2008). A esa doble vulneración, se añade la desintegración de sus redes sociales, comunitarias y culturales de 
apoyo, conformando un «triple proceso de discriminación por ser mujeres, por haber sido desplazadas, y por pertenecer a grupos étnicos».

\subsection{Múltiple violencia: física, sexual y psicológica}

El que puede ser considerado uno de los problemas más graves, en el marco de violaciones sistemáticas de derechos humanos es, en definitiva, el flagelo de la violencia sexual, física y psicológica. Según la Comisión Interamericana de Derechos Humanos (2017, 17 de abril, p. 67), «las mujeres indígenas son objeto de esclavitud sexual, embarazos forzados, violación en grupo, mutilación sexual y asesinatos a manos de diversos participantes en el conflicto armado». Asimismo, «los agentes armados usan la violencia sexual contra las mujeres indígenas como arma de guerra para desplazar comunidades por la fuerza o erosionar su capacidad de resistencia» (p. 68).

El uso de la violencia sexual en el conflicto vulnera de manera contundente la integridad física, psicológica y cultural de las mujeres afectadas. En efecto, las violaciones y abusos desarrollan de manera progresiva la figura de la dominación masculina, destruyen identidades tradicionales que daban importancia y relevancia a las mujeres y se configuran en una táctica de terror para amedrentar a la población. Asimismo, la Corte Constitucional, en el Auto 004 del 26 de enero de 2009, ha constatado el uso de tácticas como la «prostitución forzada, violencia sexual y enamoramiento de mujeres y jóvenes indígenas como táctica bélica, principalmente por parte de los actores armados ilegales». Esto agudiza la problemática de género en la comunidad, pues crea patrones de abusos que repercuten en las relaciones entre miembros de la comunidad indígena que luego pueden ser reproducidos.

Asimismo, las desapariciones y los asesinatos de mujeres indígenas son usados como tácticas de guerra que debilitan la cohesión y diseminan el miedo, con el fin de que la comunidad desista de sus posiciones de resistencia (Valero, 2016). El debilitamiento de sus estructuras políticas y administrativas despoja a las comunidades indígenas de instancias representativas, lo que es altamente necesario para dar a conocer los problemas que aquejan tanto a los individuos de cada comunidad como al colectivo. Muchos de esos liderazgos de resistencia son encabezados por mujeres.

Las estadísticas relacionadas con homicidios, desaparición forzada y desplazamientos entre mujeres de comunidades campesinas y tradicionales 
son aterradoras. Según la Alta Consejería Presidencial para la Equidad de la Mujer (2012), tomando como referencia el balance presentado por el Registro de Unidad de Victimas en Colombia, 46\% de los casos de desaparición forzada oficialmente reconocidos en el marco del conflicto armado hasta el 2012 corresponde a mujeres. Con respecto a los homicidios en el marco del conflicto armado, 47\% de las víctimas son mujeres. Asimismo, «de las 1497 personas asesinadas pertenecientes a grupos indígenas un $76 \%$ de las víctimas fueron mujeres» (Alta Consejería Presidencial para la Equidad de la Mujer, 2012, p. 20).

La firma del Acuerdo de Paz, desgraciadamente, no cambió esas cifras como era esperado. El número oficial de líderes y lideresas sociales y defensores de derechos humanos asesinados luego de la firma del Acuerdo sobrepasa los ochocientos (Pineda, 2019, noviembre 5), y de esos por lo menos 157 son miembros de comunidades indígenas (González, 2019, octubre 12). El Comité para la Eliminación de la Discriminación Racial de la Oficina del Alto Comisionado para los Derechos Humanos de las Naciones Unidas (CERD, 2019, 12 de diciembre) alertó al Estado colombiano sobre las incursiones paramilitares en territorios habitados por pueblos indígenas y comunidades afrodescendientes, acerca de los homicidios selectivos y del aumento de desplazamientos internos forzados y masivos. El Comité indicó su preocupación por las múltiples formas de discriminación que sufren las mujeres indígenas y afrocolombianas en comparación con otras mujeres del país, resaltando que: «los altos índices de violencia sexual que afecta a las mujeres indígenas y afrodescendientes de manera desproporcionada, en particular en relación con el conflicto armado, así como la falta de asistencia, protección y justicia para las víctimas, y los altos niveles de impunidad de esos crímenes» (p. 6).

\subsection{Afectación a los derechos culturales}

Además de las evidentes consecuencias en la afectación en la esfera personal y la dignidad humana de las mujeres indígenas, el conflicto armado trae una importante transgresión de los derechos colectivos, estilo de vida y cosmovisión de las comunidades indígenas y tribales. Las experiencias extremas con la violencia física inciden en sus estructuras culturales y generan una modificación dañina de los roles de la mujer en la reproducción de la cultura en la comunidad y, por ende, en la garantía de la permanencia de sus pueblos. 
Tener que dejar los territorios ancestrales genera un impacto cultural irreparable. El fenómeno del desplazamiento forzado transgrede sustancialmente las formas tradicionales y modelos de vida de las comunidades, y en especial de las mujeres indígenas. En el documento Uguerara Da Amba Giunu Tabua entregado el 20 de junio de 2005 por el área Mujer de la Organización de las Nacionalidades Indígenas de Colombia (ONIC) a la Relatora de la $\mathrm{CIDH}$, las mujeres indígenas manifestaron que:

$\mathrm{Si}$ es un desplazamiento interno se presentan problemas porque están rompiendo con el equilibrio de nuestros ecosistemas. Si es a centros urbanos se complica un poco más, ya que la mayoría de nuestras compañeras no saben muy bien el español, se asustan ante la inmensidad de la ciudad, el anonimato y la falta de solidaridad de sus habitantes, nos mata el recuerdo de nuestros montes y selvas y de sus sonidos. Nos enfrentamos a nuevos problemas para criar a nuestros hijos y relacionarnos con nuestros compañeros, por no ser la ciudad nuestro medio habitual. Nos persiguen las imágenes de la angustia cuando tuvimos que salir corriendo con lo poco que teníamos o podíamos cargar para hacerle el quite a la muerte y desolación, en medio de esta angustia, quedamos a cargo de la familia aceptando actividades que no son tradicionales a nuestras culturas como emplearnos en el trabajo doméstico, o en el peor de los casos llegar a vender nuestros cuerpos (CIDH, 2006, 18 de octubre b).

De acuerdo con el Centro Nacional de Memoria Histórica y la Organización Nacional Indígena de Colombia (2019) existen tres procesos con que los pueblos indígenas de Colombia entienden el conflicto: interrupción, alteración y administración de la vida. El proceso de interrupción de la vida se traduce generalmente en acción violenta que provoca la mala muerte. Los procesos de alteración abarcan las violencias no letales que inciden en «la posibilidad de circulación de la vida en las redes viales del territorio»y, por último, el conflicto afecta la administración de la vida porque impone formas ajenas de administrar los territorios que reorientan la vida de los territorios indígenas por fuera de la Ley de origen.

Todas las afectaciones a los llamados tejidos de vida de los pueblos indígenas y otras comunidades étnicas deben ser tenidas en cuenta en el momento de diseñar y aplicar las políticas públicas pensadas en la situación de posconflicto. Es un gran desafío para un Estado centralizado como Colombia responder a violaciones masivas de mujeres indígenas cuyo modo 
y perspectiva de vida generalmente no es contemplado por los gobiernos nacionales.

\section{La aplicación de políticas públicas adecuadas a la realidad de las mujeres indígenas}

La CIDH (2018, 15 de septiembre) define una política pública como «los lineamientos o cursos de acción que definen las autoridades de los Estados para alcanzar un objetivo determinado, y que contribuya a crear o transformar las condiciones en que se desarrollan las actividades de los individuos o grupos que integran la sociedad» (p. 45).

La adopción de políticas públicas supone un esfuerzo estatal en el que convergen metodologías de identificación de problemas sociales, planes de acción y disponibilidad presupuestal. Y si es entendida desde un enfoque de derechos humanos, esos son el núcleo y la razón de ser de la acción del Estado. Tanto las obligaciones asumidas por el Estado en materia de derechos humanos, como las vulnerabilidades sociales de cada país deben ser identificadas en el momento de concebir una política pública. Avanzar en una política pública con enfoque basado en derechos humanos implica

[ 78 ] por lo menos estos cuatro pasos: a) identificación de la situación a atender; b) diseño o formulación de la política; c) implementación o ejecución de la política; d) monitoreo y evaluación (CIDH, 2018, 15 de septiembre).

Para diseñar una política pública con enfoque de género es necesario identificar no solo las causas y consecuencias de las desigualdades de géneros, sino además tener en cuenta las diferencias socioeconómicas y culturales que existen entre las mujeres (Valle y Bueno, 2006). La articulación de componentes políticos, económicos y culturales que están involucrados en la vulnerabilidad de determinado grupo es lo que da protagonismo al enfoque transversal de género, desarrollado en la Tercera Conferencia Internacional sobre la Condición Jurídica y Social de la Mujer, en Nairobi, 1985. La característica principal de este enfoque es la inclusión de la mujer en el proceso de formulación y aplicación de las políticas públicas que las afectan (Zaremberg, 2007).

La transversalidad también invita a generar espacios interseccionales dentro de las políticas públicas, permitiendo tener una visión más abarcadora de la opresión enfrentada por cada grupo (Coll-Planas, Solá- 
Morales y Missé, 2019). Como se indicó, las mujeres indígenas enfrentan una triple discriminación, siendo esa vulnerabilidad múltiple lo que exige un entendimiento holístico de las causas de esas vulnerabilidades. Tratar de manera separada los motivos que generan cada eje de discriminación no garantiza la eliminación de las causales de la triple vulnerabilidad, pues cada grupo está afectado de manera distinta por cada eje, y cada afectación, a su vez, influye la posición de vulnerabilidad del grupo con relación a otro eje (Incháustegui y Ugalde, 2004).

Al reconocer la transversalidad e interseccionalidad de los factores que componen determinada situación de vulnerabilidad, se comprende la importancia central de la participación de los grupos vulnerables destinatarios de políticas públicas en su diseño e implementación. En el caso de las mujeres indígenas esa participación debe darse según lo establecido en los compromisos del Estado colombiano ante la comunidad internacional, es decir, con base en el respeto y la protección de la autodeterminación de los pueblos indígenas y propiciando la participación de esos pueblos en las políticas públicas por medio de la consulta previa. Las políticas públicas deben incluir medidas positivas que abarquen la perspectiva de las mujeres y medidas que les permitan sobrepasar las enormes dificultades que existen para que sus voces sean escuchadas ( $\mathrm{CIDH}, 2017,17$ de abril). Esas medidas deben siempre ser consultadas y diseñadas con ellas.

\section{El enfoque diferencial: una herramienta de políticas públicas}

Esa necesidad de analizar a los sujetos de políticas públicas desde otra perspectiva e implementar medidas concebidas a partir de un enfoque no tradicional fue traducida, en Colombia, por la noción de enfoque diferencial. No existe claridad jurídica o conceptual respecto al significado del enfoque diferencial en Colombia. De acuerdo con la Oficina del Alto Comisionado para los Derechos Humanos de las Naciones Unidas (s. f.), el enfoque diferencial es un «método de análisis» que quiere visibilizar las formas de discriminación hacia grupos considerados diferentes a la sociedad hegemónica. El Ministerio de Salud y Protección Social (2013, noviembre 14) también considera el enfoque diferencial como un método de análisis, actuación y evaluación para cualificar la respuesta del Estado en la garantía de derechos. El enfoque diferencial debe involucrar «las condiciones y posiciones de los/las distintos/ as actores sociales como sujetos/as de derecho, desde una mirada de grupo 
socioeconómico, género, etnia e identidad cultural, y de las variables implícitas en el ciclo vital - niñez, juventud, adultez y vejez».

Para la Secretaría Distrital de Integración Social de Bogotá (s. f.), el enfoque diferencial identifica las características que comparten las personas con el objetivo de potenciar las acciones diferenciales que materialicen el goce efectivo de sus derechos. Para el Ministerio del Interior (2015), el enfoque diferencial «puede entenderse como el conjunto de acciones que, al dar un trato diferenciado a algunos grupos poblacionales, contribuye a reducir la brecha existente entre los diferentes segmentos de la población» (p. 7). Esa definición puede ser problematizada cuando ese enfoque pretende ser aplicado en sociedades tan diversas - y no hegemónicas—como la colombiana, pero esa discusión sale del ámbito de este artículo.

En la Corte Constitucional de Colombia el enfoque diferencial adquirió protagonismo a inicios del milenio. Luego de que se presentaran múltiples acciones de tutela por parte de diferentes ciudadanos desplazados y ante la negligencia del Estado para contrarrestar las nefastas consecuencias del desplazamiento forzado, la Corte Constitucional (Sentencia T-602/03 del 23 de junio de 2003) instó al Estado a desarrollar políticas públicas de

[ 80 ] salvaguarda de los derechos fundamentales que se ven violados cuando una comunidad es despojada de su territorio. En aquella ocasión, la Corte enfatizó la necesidad de que la atención a la población desplazada se fundara en acciones afirmativas y en enfoques sensibles al género, a la generación, a la etnia, a la discapacidad y a la orientación sexual. En este sentido, insistió la Corte, las medidas positivas debían tener como norte la satisfacción de los requerimientos de aquellos grupos poblacionales colocados en especial situación de indefensión. La Corte precisó que la acción afirmativa «se justifica en que resulta indispensable todo un conjunto de actos de política pública con el objeto de lograr la igualdad material de los desplazados».

Posteriormente, cuando la Corte Constitucional declaró el estado de cosas inconstitucional (Quintero, Navarro y Meza, 2011, pp. 71-72) en la situación de los desplazados (Sentencia T-025/04 del 22 de enero de 2004), señalando la falta de concordancia entre, por un lado, la gravedad de la afectación de los derechos humanos y, por otro, los recursos disponibles y la capacidad institucional para para asegurar el goce efectivo de tales derechos, esto la llevó a establecer distintos mandatos a las entidades estatales encargadas 
de proteger los derechos vulnerados de toda la comunidad desplazada. A pesar de que en la referida sentencia aún no existía una definición precisa sobre el significado de enfoque diferencial, este fallo generó diferentes autos de seguimiento que posteriormente fueron conocidos como «autos de enfoque diferencial».

Entre esos autos de seguimiento, la Corte manifiesta que el derecho internacional requiere que las autoridades colombianas adopten «un enfoque de prevención del desplazamiento forzado que sea lo suficientemente diferenciado como para incidir sobre las causas de fondo de este fenómeno y su impacto desproporcionado sobre los derechos humanos de las mujeres» (Auto 092 del 14 de abril de 2008). Nótese que «suficientemente diferenciado» se refiere a la vulnerabilidad de las víctimas, mientras que las causas de fondo de los desplazamientos muy poco o nada tiene que ver con ellas.

La Corte también estableció una serie de medidas especiales para ser adoptadas por parte del Gobierno nacional en la realización de los derechos de los pueblos indígenas, instando al Estado a diseñar y a implementar, en consulta con las organizaciones indígenas del país, un programa de garantía de los derechos de los pueblos indígenas afectados por el desplazamiento (Auto 004 del 29 de enero de 2009). En 2010, la Corte indicó como debe ser diseñado un enfoque diferencial en la aplicación de políticas públicas:

El diseño de un enfoque diferencial requiere identificar las diferencias de los grupos de especial atención en razón de su mayor vulnerabilidad, como en este caso son los indígenas, para determinar cuáles diferencias son relevantes y encaminar cambios en la política, que son posibles si se realiza un ejercicio de análisis en el que se identifiquen los vacíos existentes en la respuesta estatal, así como las transformaciones para ajustar, modificar o complementar la política, llenándola de contenido propio y coherente a la atención de esta población y con la obligación de asegurar el goce efectivo de sus derechos (Auto 382 del 10 de diciembre de 2010).

Desde esa perspectiva, puede parecer que se sugiere que algunos agentes del Estado determinen cuáles diferencias de determinado grupo vulnerables son relevantes - y cuáles no- para la aplicación de políticas diferenciadas. Aunque en esta ocasión la Corte no lo resaltó, esa determinación debe ser hecha con la participación de los grupos destinatarios. 
En 2012, la Corte Constitucional finalmente ofreció una definición más precisa de la noción de enfoque diferencial, que «se traduce en la adopción de una serie de medidas encaminadas a enfrentar la situación de vulnerabilidad acentuada de algunas víctimas en razón de su edad, género, orientación sexual y situación de discapacidad» (Sentencia C-253A del 29 de marzo de 2012). Para la Corte, las leyes deben contribuir a eliminar los esquemas de discriminación y marginación y ofrecer medidas de protección, asistencia y reparación «a los miembros de grupos expuestos a mayor riesgo de violaciones de sus derechos fundamentales: mujeres, jóvenes, niños y niñas, personas mayores, personas en situación de discapacidad, líderes sociales, miembros de organizaciones sindicales, defensores de derechos humanos y víctimas de desplazamiento forzado».

Ese entendimiento de la Corte se afina con el artículo $1 .^{\circ}$ de la Convención Internacional de las Naciones Unidas para la Eliminación de todas las Formas de Discriminación Racial (Resolución 2106A (XX) del 21 de diciembre de 1965), que exige que los Estados parte implementen medidas especiales que faciliten que las poblaciones más vulnerables logren condiciones de igualdad. Para evitar la terminología de «discriminación positiva», que iría en contra de los principios de la Convención -y que anteriormente fue utilizada por la Corte Constitucional colombiana supra-, el CERD (2009, 29 de septiembre) recomendó a los Estados utilizar términos que reflejen más claramente las obligaciones asumidas en la Convención:

El término «medidas especiales» comprende medidas que en algunos países podrían denominarse «acción afirmativa», «medidas afirmativas» o «acción positiva» [...]. Al igual que la Convención, la presente recomendación emplea los términos «medidas especiales» $y$ «medidas especiales y concretas» y alienta a los Estados parte a emplear una terminología que refleje claramente la relación entre sus leyes y prácticas y estos conceptos enunciados en la Convención. El término «discriminación positiva» es contradictorio en el contexto de las normas internacionales de derechos humanos y debe evitarse.

\subsection{La aplicación del enfoque diferencial en la Ley de Víctimas}

En 2011 el Congreso colombiano aprobó la Ley de Víctimas y Reparación de Tierras (Ley 1448 del 10 de junio de 2011), estableciendo 
«un conjunto de medidas judiciales, administrativas, sociales y económicas, individuales y colectivas, en beneficio de las violaciones contempladas [...] en un marco de justicia transicional». Pese a que no existieran dudas de que esa Ley afectaría los derechos de los pueblos indígenas víctimas del conflicto armado, las organizaciones indígenas no fueron previamente consultadas sobre su contenido.

Por exigencia de representantes indígenas de la Mesa Permanente de Concertación con los Pueblos y Organizaciones Indígenas, y para evitar que la Ley fuera demandada por inconstitucionalidad (Aponte y López, 2013), las disposiciones transitorias de la Ley otorgan poderes especiales al presidente de la República para expedir decretos con fuerza de ley con el fin de regularizar «los derechos y garantías de las víctimas pertenecientes a pueblos y comunidades indígenas, ROM y negras, afrocolombianas, raizales y palenqueras» en su aplicación (Ley 1448 del 10 de junio de 2011).

Durante el primer debate del Proyecto de Ley de Víctimas en el Congreso se señaló que el otorgamiento de facultades extraordinarias al presidente tenía como finalidad «abordar la situación de vulnerabilidad acentuada en que se encuentran [las víctimas], atendiendo a sus necesidades específicas en virtud del principio del enfoque diferencial» (Aponte y López, 2013, p. 167).

El Decreto Ley 4633 del 9 de diciembre de 2011, que materializó las facultades extraordinarias del presidente, sí fue presentado a algunos representantes de organizaciones indígenas. Debido a limitaciones de tiempo y la premura de su publicación, muy pocos líderes o lideresas indígenas conocieron el tenor del Decreto antes de su vigencia. En pocas palabras, ni la Ley de Víctimas, ni el Decreto Ley fueron consultados en los términos de la ley doméstica e internacional que trata sobre la consulta previa. Sin embargo, ambos son los instrumentos jurídicos que establecen los lineamientos respecto a la noción de enfoque diferencial para reparación de mujeres indígenas.

Una de las medidas —llamada «acción afirmativa»— previstas por el Decreto es priorización de grupos vulnerables, entre ellos las mujeres indígenas, «en la atención, asistencia y reparación integral». Asimismo, el Decreto Ley 4633 de 2011 pretende que las medidas previstas contribuyan «a la eliminación de las estructuras de discriminación, exclusión y marginación» (art. 15). Un reflejo de falta de claridad sobre lo que es una medida de acción 
afirmativa, o de enfoque diferencial, es que se pretenda que tales medidas incidan sobre las estructuras de las desigualdades, mientras que su finalidad es que las medidas de reparación - y no estructurales - sean aplicadas de manera adecuada a la situación social de la víctima.

\subsection{La aplicación del enfoque diferencial en el Acuerdo de Paz}

Otro ejemplo importante de aplicación de esa herramienta es lo plasmado en el Acuerdo de Paz entre el Gobierno de Colombia y FARC-EP, el cual prevé la implementación de un enfoque no solamente de género, sino también territorial y diferencial:

El Acuerdo está compuesto de una serie de acuerdos, que sin embargo constituyen un todo indisoluble, porque están permeados por un mismo enfoque de derechos, para que las medidas aquí acordadas contribuyan a la materialización de los derechos constitucionales de los colombianos; por un mismo enfoque diferencial y de género, para asegurar que la implementación se haga teniendo en cuenta la diversidad de género, étnica y cultural, y que se adopten medidas para las poblaciones y los colectivos más humildes y más vulnerables, en especial los niños y las niñas, las mujeres, las personas en condición de discapacidad y las víctimas; y en especial por un mismo enfoque territorial (Gobierno de Colombia y FARC-EP, 2016, p. 4).

Pese que el Acuerdo no ofrece una definición para cada enfoque, infiere que su objetivo es la protección del principio de la no discriminación e igualdad en el pluralismo; y que una de sus herramientas es la participación (Gobierno de Colombia y FARC-EP, 2016). Asimismo, el Acuerdo hace referencias relacionadas con la implementación de algún tipo de enfoque diferencial para determinadas víctimas del conflicto. El Acuerdo establece que el Programa Nacional Integral de Sustitución de Cultivos de Uso llícito (PNIS) sea implementado con un «enfoque territorial y de género», es decir, que debe «tener en cuenta las necesidades, características y particularidades económicas, culturales y sociales de los territorios y las comunidades rurales, en especial de las comunidades indígenas y afrodescendientes, y de las mujeres en estas comunidades y territorios» (Gobierno de Colombia y FARC$E P, 2016$, p. 6). Sobre el enfoque de género relacionado al consumo de drogas, el Acuerdo recuerda la necesidad de que se identifiquen: 
El marco jurídico del enfoque diferencial en políticas públicas para mujeres indígenas...

Factores de vulnerabilidad asociados a edad, sexo, condición de discapacidad, condición socioeconómica y ubicación geográfica o pertenencia a la población LGBTI, entre otros.

[...] Este enfoque deberá tener en cuenta la relación entre el consumo de las drogas ilícitas y la violencia contra la mujer, especialmente con la violencia intrafamiliar y la violencia sexual. Se preverán medidas para mujeres, niñas, jóvenes y adolescentes. En todo caso, las acciones que se adelanten respetarán el uso ancestral de la hoja de coca por parte de las comunidades indígenas (Gobierno de Colombia y FARCEP, 2016, pp. 47 y 117).

Sin realizar una conceptualización jurídica del enfoque diferencial, el Acuerdo lo presenta como un mero mecanismo de implementación, vacío de contenido procesal. Es importante resaltar que el Acuerdo no incluye un enfoque de género en el llamado enfoque territorial, ni tampoco establece medidas especiales de protección a las sexualidades diversas en la implementación de las medidas relacionadas con pueblos étnicos.

En el marco de las negociaciones de paz entre el Gobierno de Colombia y FARC-EP se acordó constituir una subcomisión de género integrada por miembros del Gobierno nacional, las FARC-EP, mujeres víctimas del conflicto armado, mujeres campesinas, mujeres indígenas y afrodescendientes. Aunque reconoce la importancia de la equidad de género como pilar fundamental para alcanzar la paz, el Acuerdo sin embargo solo trata la problemática de la mujer indígena junto con la problemática de la mujer en general, desconociendo su situación de vulnerabilidad específica, que incluye la pérdida de identidad cultural y colectiva a raíz del conflicto, la importancia del territorio y la desidia administrativa que las ha condenado desde la colonización española.

\section{Análisis de la efectividad del enfoque diferencial como medida especial y transitoria de protección}

El enfoque diferencial es presentado, tanto en los Acuerdos de Paz como en la legislación y la jurisprudencia colombiana, como una herramienta para la protección y salvaguarda de los derechos de las comunidades indígenas y tribales, con especial énfasis en grupos que poseen triple vulnerabilidad: las mujeres indígenas. Es voz unánime en las instancias gubernamentales que, dadas las condiciones planteadas, es necesario que todos los procesos contengan medidas que impulsen condiciones de equidad entre poblaciones 
con características diferentes. El CERD (2009, 29 de septiembre, p. 5) indica cuatro condiciones de aplicación de esas medidas: a) adecuadas a la situación que quiere remediarse; b) legítimas; c) necesarias en una sociedad democrática; d) respetar los principios de justicia y proporcionalidad. Además, las necesidades de esas medidas deben ser evaluadas sobre la base de «datos precisos, desglosados por raza, color, linaje y origen étnico o nacional y que incorporen una perspectiva de género», asegurándose de que las comunidades beneficiarias sean consultadas respecto a su aplicación.

Según la Convención Internacional sobre la Eliminación de todas las Formas de Discriminación Racial (Asamblea General de las Naciones Unidas, Resolución 2106A (xx) del 21 de diciembre de 1965) esas medidas deben ser especiales, temporales y «destinadas a garantizar el disfrute pleno e igual de los derechos humanos y las libertades fundamentales por los grupos desfavorecidos». Tales medidas especiales no deben ser confundidas con los derechos permanentes que son reconocidos en los instrumentos de derechos humanos.

Colombia es un Estado parte de la Convención Internacional de las Naciones Unidas para la Eliminación de todas las Formas de Discriminación

[ 86 ] Racial, ratificando la convención en 1981. En virtud de las obligaciones internacionales que la ratificación acarrea, es menester que el Estado aplique dichas disposiciones a fin de generar acciones especiales para salvaguardar efectivamente los derechos de poblaciones vulnerables en razón de etnia.

La consulta previa difiere de las medidas especiales, porque estas deben tener como finalidad facilitar y promover la realización de los derechos humanos reconocidos a los grupos históricamente discriminados, pero no deben confundirse con los derechos humanos que pretenden garantizar. De hecho, la manera idónea de comprender la perspectiva de las mujeres indígenas y construir medidas efectivas de reparación y no repetición es la consulta previa. Es con el fin de construir ese canal de diálogo y de intercambio de perspectivas que el Estado tiene el deber de consultar con los pueblos indígenas antes de implementar cualquier política que afecte el ejercicio de sus derechos.

Si bien las medidas especiales, como el enfoque diferencial, pueden y deben ser utilizadas en la aplicación de políticas públicas con el fin de 
lograr una equidad en situaciones asimétricas, estas no pueden ser utilizadas para evadir obligaciones estatales. Cualquier medida especial de enfoque diferencial debe ser, también, consultada con su destinatario, evitando intervenciones arbitrarias por parte del Estado. Es precisamente esto lo que no está sucediendo en las políticas públicas enfocadas en superar las vulnerabilidades de las mujeres indígenas en Colombia.

Como se observó, la garantía de la efectividad de las políticas públicas va de la mano de la participación. La falta de participación de los destinatarios de políticas públicas en su concepción, diseño e implementación no puede ser reemplazada por ninguno de los mecanismos del llamado enfoque diferencial.

\section{Conclusión}

La situación del Estado colombiano respecto a la implementación de políticas públicas con perspectivas étnicas y de género no ha incluido de manera efectiva y en los parámetros de los derechos humanos la participación de mujeres indígenas en su planificación y vigilancia. El Estado no ha desarrollado una metodología que contenga datos precisos, desglosados por diferencias culturales de origen étnico o nacional, y que incorporen una perspectiva de género, tal como requiere el derecho internacional y los derechos humanos.

La restitución de tierras es uno de los puntos más álgidos y, por lo tanto, más complejos para desenvolver dentro de las políticas públicas de posconflicto. Políticas públicas para pueblos indígenas deben ser siempre consultadas de manera previa, según los parámetros del derecho doméstico e internacional. Obviar la consulta y privilegiar el uso del enfoque diferencial trae el riesgo de que las decisiones sobre políticas públicas para mujeres indígenas víctimas de conflicto permanezca en las manos del Estado, tal como ocurrió con el Decreto Ley 4633 de 2011. Finalmente, no sobra recalcar la urgente misión del Estado colombiano de velar por la protección física de lideresas indígenas que son amenazadas física, psicológica y sexualmente en la lucha por sus derechos. Esa es la única manera de demostrar la buena fe que es la base de cualquier relación social, especialmente en situación de posconflicto y conciliación. 


\section{Referencias bibliográficas}

1. Alta Consejería Presidencial para la Equidad de la Mujer. (2012). Lineamientos de la política pública nacional de equidad de género para las mujeres. Recuperado de http://www.equidadmujer.gov.co/Documents/Lineamientos-politica-publicaequidad-de-genero.pdf

2. Aponte, José Martín y López, Lina María. (2013). El pluralismo jurídico indígena en Ley de Víctimas y Restitución de Tierras: Retorno y consulta previa. Universitas Estudiantes, 10, pp. 157-176. Recuperado de https://cienciasjuridicas.javeriana.edu. co/documents/3722972/4350738/8 + el + pluralismo + jur\%C3\%ADdico + 157-176. pdf/14f6a3e5-d474-44d3-b40d-0485fe3b8e8a

3. Asamblea General de las Naciones Unidas. Resolución 2106A (xx). (21 de diciembre de 1965). Convención Internacional sobre la Eliminación de todas las Formas de Discriminación Racial. Recuperado de https://www.ohchr.org/SP/ Professionallnterest/Pages/CERD.aspx

4. Centro Nacional de Memoria Histórica y Organización Nacional Indígena de Colombia. (2019). Tiempos de vida y muerte. Memorias y luchas de los Pueblos Indígenas en Colombia. Bogotá, D. C.: CNMH-ONIC.

5. Coll-Planas, Gerard; Solá-Morales, Rose y Missé, Miquel. (2019). Interseccionalidad en las políticas LGBTI metropolitanas. Montevideo: Metrópolis.

6. Colombia. Congreso de la República. Ley 1448. (10 de junio de 2011). Por la

[ 88 ] cual se dictan medidas de atención, asistencia y reparación integral a las víctimas del conflicto armado interno y se dictan otras disposiciones. Recuperado de http://www. secretariasenado.gov.co/senado/basedoc/ley_1448_2011.html

7. Colombia. Corte Constitucional. Sentencia T-602/03. (23 de julio de 2003). Recuperado de https://www.corteconstitucional.gov.co/relatoria/2003/T-602-03.htm

8. Colombia. Corte Constitucional. Sentencia T-025/04. (22 de enero de 2004). Recuperado de https://www.corteconstitucional.gov.co/relatoria/2004/t-025-04.htm

9. Colombia. Corte Constitucional. Auto 092. (14 de abril de 2008). Recuperado de https://www.corteconstitucional.gov.co/relatoria/autos/2008/a092-08.htm

10. Colombia. Corte Constitucional. Auto 004. (29 de enero de 2009). Recuperado de https://www.corteconstitucional.gov.co/T-025-04/AUTOS\%202009/111.\%20 Auto \%20del\%2026-01-2009.\%20Auto\%20004.\%20Indigenas.pdf

11. Colombia. Corte Constitucional. Auto 382. (10 de diciembre de 2010). Recuperado de https://www.corteconstitucional.gov.co/relatoria/autos/2010/A38210.htm

12. Colombia. Corte Constitucional. Sentencia C-253A. (29 de marzo de 2012). Recuperado de https://www.corteconstitucional.gov.co/relatoria/2012/C-253A-12. htm

13. Colombia. Ministerio del Interior. Decreto Ley 4633. (9 de diciembre de 2011). Por medio del cual se dictan medidas de asistencia, atención, reparación 
El marco jurídico del enfoque diferencial en políticas públicas para mujeres indígenas...

integral y de restitución de derechos territoriales a las víctimas pertenecientes a los pueblos y comunidades indígenas. Recuperado de https://www.mininterior.gov.co/ sites/default/files/Gactv/Normatividad/decreto_4633_de_2011.pdf

14. Comisión Interamericana de Derechos Humanos (CIDH). (2006, 18 de octubre a). Las mujeres frente a la violencia y la discriminación derivadas del conflicto armado en Colombia. Recuperado de http://www.cidh.org/countryrep/ColombiaMujeres06sp/ Informe \% 20Mujeres \%20Colombia\%202006\%20Espanol.pdf

15. Comisión Interamericana de Derechos Humanos (CIDH). (2006, 18 de octubre b). La múltiple discriminación contra las mujeres afrocolombianas e indígenas. En: Las mujeres frente a la violencia y la discriminación derivadas del conflicto armado en Colombia. Recuperado de http://www.cidh.org/countryrep/colombiamujeres06sp/ IV.htm\#_ftn171

16. Comisión Interamericana de Derechos Humanos (CIDH). (2011, 28 de diciembre). Acceso a la justicia para mujeres víctimas de violencia sexual: la educación y la salud. Recuperado de https:/www.oas.org/es/cidh/mujeres/docs/pdf/ VIOLENCIASEXUALEducySalud.pdf

17. Comisión Interamericana de Derechos Humanos (CIDH). (2011, 31 de diciembre). Segundo informe sobre la situación de las defensoras y defensores de los derechos humanos en las Américas. Recuperado de https://www.oas.org/es/cidh/ defensores/docs/pdf/defensores2011.pdf

18. Comisión Interamericana de Derechos Humanos (CIDH). (2017, 17 de abril). Las mujeres indígenas y sus derechos humanos en las Américas. Recuperado de http:// www.oas.org/es/cidh/informes/pdfs/MujeresIndigenas.pdf

19. Comisión Interamericana de Derechos Humanos (CIDH). (2018, 15 de septiembre). Políticas públicas con enfoque de derechos humanos. Recuperado de https://www.oas.org/es/cidh/informes/pdfs/PoliticasPublicasDDHH.pdf

20. Comité para la Eliminación de la Discriminación Racial (CERD). (2009, 29 de septiembre). Significado y alcance de las medidas especiales en la Convención Internacional sobre la Eliminación de todas las Formas de Discriminación Racial. Acnur. Recuperado de https://www.acnur.org/fileadmin/Documentos/BDL/2012/8464.pdf

21. Comité para la Eliminación de la Discriminación Racial (CERD). (2019, 12 de diciembre). Observaciones finales sobre los informes periódicos $17^{\circ}$ a $19^{\circ}$ combinados de Colombia. OHCHR. Recuperado de https://tbinternet.ohchr.org/Treaties/CERD/ Shared\%20Documents/COL/INT_CERD_COC_COL_40807_S.pdf

22. Gobierno de Colombia y FARC-EP. (2016). Acuerdo final para la terminación del conflicto y la construcción de una paz estable y duradera. Alto Comisionado para la Paz. Recuperado de http://www.altocomisionadoparalapaz.gov.co/procesosy-conversaciones/Documentos\%20compartidos/24-11-2016NuevoAcuerdoFinal.pdf

23. González, Leonardo. (2019, octubre 12). Que pare el genocidio. Indepaz. Recuperado de http://www.indepaz.org.co/wp-content/uploads/2019/10/Doc.ind\%C3\%ADgenas12-10-2019.pdf 
24. Incháustegui, Teresa y Ugalde, Yamileth. (2004). Materiales y herramientas conceptuales para la transversalidad de género. México, D. F.: Inmujeres.

25 inisterio de Salud y Protección Social. (2013, noviembre 14). Enfoque diferencial. Recuperado de https://www.minsalud.gov.co/Lists/Glosario/DispForm. aspx? ID = 21

26. Ministerio de Salud y Protección Social. (2016). Perfil de salud de la población indígena, y medición de desigualdades en salud. Colombia 2016. Recuperado de https://www.minsalud.gov.co/sites/rid/Lists/BibliotecaDigital/RIDE/VS/ED/PSP/Perfilsalud-pueblos-indigenas-colombia-2016.pdf

27. Ministerio del Interior. (2015). El enfoque diferencial y étnico en la política de víctimas del conflicto armado. Bogotá, D. C.: Ministerio del Interior. Recuperado de https://gapv.mininterior.gov.co/sites/default/files/cartilla_enfoque diferencial_fin_1.pdf

28. Observatorio de Asuntos de Género. Presidencia de la República. (2013). Algunos factores asociados a la violencia contra las mujeres indígenas, Colombia 2012-2013 (estudio exploratorio). Recuperado de http://www.equidadmujer.gov.co/ oag/Documents/Investigacion_indigena.pdf

29. Oficina del Alto Comisionado para los Derechos Humanos de las Naciones Unidas. (s. f.). ¿Qué es el enfoque diferencial? Recuperado de https://www.hchr.org. co/index.php/76-boletin/recursos/2470-ique-es-el-enfoque-diferencial

30. Pineda, Luz Bibiana. (2019, noviembre 5). 800 asesinatos, 800 razones. El País. Recuperado de https://elpais.com/elpais/2019/10/29/ migrados/1572368189_343900.html

31. Quintero, Josefina; Navarro, Angélica y Meza, Malka. (2011). La figura del estado de cosas inconstitucionales como mecanismo de protección de los derechos fundamentales de la población vulnerable en Colombia. Revista Jurídica Mario Alario D'Filippo, 3 (1), pp. 70-81. Recuperado de https://dialnet.unirioja.es/servlet/ articulo?codigo $=4767667$

32. Secretaría Distrital de Integración Social. (s. f.). Enfoque diferencial. Recuperado de http://www.integracionsocial.gov.co/index.php/politicas-publicas/lasdis-aporta-a-la-implementacion/politica-publica-enfoque-diferencial

33. Valero, Ana. (2016). Violencia y resistencia: mujeres indígenas desplazadas en Colombia. Lectora, 22, pp. 43-52. Recuperado de http://revistes.ub.edu/index.php/ lectora/article/view/Lectora2016.22.4

34. Valle, Gloria y Bueno, Eramis. (2006). Las políticas públicas desde una perspectiva de género. Novedades en Población, 2 (4). Recuperado de http://www. novpob.uh.cu/index.php/NovPob/article/view/87/119

35. Zaremberg, Gisela (ed.). (2007). Políticas sociales y género: Los problemas sociales y metodológicos. México, D. F.: Flacso. 